\title{
Filtering of the skin portion on lung ultrasound digital images to facilitate automatic diagnostics of pneumonia
}

\author{
Franklin Barrientos ${ }^{1}$, Avid Roman-Gonzalez ${ }^{1}$, Ronald Barrientos ${ }^{1}$, Leonardo Solis ${ }^{1}$, Alicia Alva ${ }^{1}$, Malena \\ Correa $^{1}$, Monica Pajuelo ${ }^{1}$, Cynthia Anticona ${ }^{1}$, Roberto Lavarello ${ }^{2}$, Benjamin Castañeda ${ }^{2}$, Richard Oberhelman $^{3}$, \\ Robert H. Gilman ${ }^{4}$, Mirko Zimic ${ }^{1 *}$ \\ ${ }^{1}$ Laboratorio de Bioinformática y Biología Molecular, Facultad de Ciencias, Universidad Peruana Cayetano Heredia -
}

$\mathrm{UPCH}$

${ }^{2}$ Laboratorio de Imágenes Médicas, Departamento de Ingeniería, Pontificia Universidad Catolica del Peru - PUCP

${ }^{3}$ Tulane University, School of Public Health and Tropical Medicine - TU

${ }^{4}$ Department of International Health, School of Public Health, Johns Hopkins University - JHU

*mirko.zimic@upch.pe

\begin{abstract}
Pneumonia is one of the major causes of child mortality, but it is curable if one can achieves early diagnostics. Unfortunately, in developing countries there is a lack of infrastructure and medical experts in rural areas to provide the required diagnostics opportunely. Lung ultrasound echography has proved to be an important tool to detect lung consolidates as evidence of pneumonia. The use of ultrasound to detect pneumonia is limited by the image analysis for interpretation, which is carried by human experts. Pattern recognition and image analysis is a potential tool to facilitate recognition of pneumonia consolidates in absence of medical experts for automatic diagnostics. To perform an automatic analysis of lung ultrasound images for pneumonia detection, the noise introduced by the image portion of the skin, notably complicates the processing and interpretation. This paper presents a methodology to recognize and eliminate the portion of the skin in lung ultrasound images.
\end{abstract}

Keywords - Ultrasound images, echography, image processing, remote diagnostics.

\section{INTRODUCTIÓN}

$\mathrm{P}$ NEUMONIA is the world's leading cause of child mortality, (1.4 million deaths/year) in children under five (UNICEF 2013).

It is usually diagnosed based on x-ray images and laboratory/clinical examination. This depends on trained medical professionals who are scarce in rural communities in developing countries.

In recent studies, thoracic echography has also been proved as an adequate approach to diagnose pneumonia in children. Unfortunately, due to its high cost, low portability, and the necessity to have a human expert to interpret the echographic images, thoracic echography is not feasible to be used in rural regions like the Andean highlands where pneumonia affects to several children.

In this study we show the first step related to image processing, of a specific algorithm of pattern recognition to detect lung consolidates as evidence of pneumonia in children.

This step includes the filtering of the skin portion in a lung echographic ultrasound digital image, to reduce noise and favor the correct interpretation for pneumonia diagnostics.

\section{Methodology: Skin Removal FiLter}

We collected lung-ultrasound images from 23 children under 5 years old (15 with diagnosis of pneumonia and 8 healthy children). These children were examined at the Hospital del Niño in Lima - Peru. Lung ultrasound images (Figure 1) were obtained from a standard medical screening using a SONIXTOUCH ultrasound system with the probe L14-5/38 (Ultrasonix, Brithish Columbia, Canada). An expert physician analyzed the recorded videos. Frames with clear evidence of pneumonia, as well as frames with no evidence of pneumonia were classified as positive or negative respectively. These frames were analyzed to filter the skin portion in order to facilitate the diagnostics of pneumonia.

The removal of skin and other elements not involving the lungs is transcendental for pneumonia identification based on ultrasound images. This is because image areas corresponding to both the epidermis and muscle tissue do not contribute to any extent the subsequent 
imaging analysis and processing. With this goal in mind, we have developed a method, which focuses on high-value pixels (values greater than 100, color depth of 8 bits in grayscale), which are concentrated in the upper region of lung ultrasound images obtained from an ultrasound scanner.

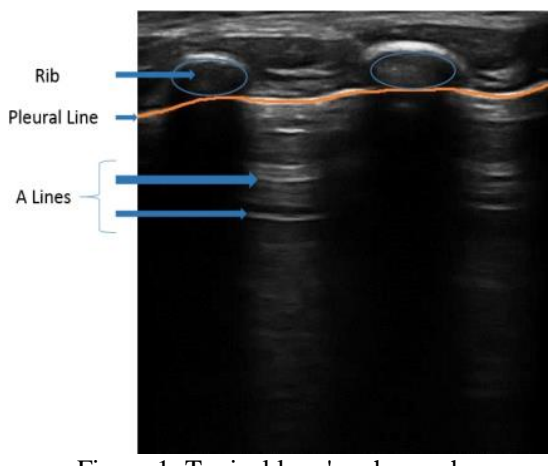

Figure 1: Typical lung's echography

The high concentration of high-value pixels in certain regions is because sound waves emitted by the ultrasound scanner are mostly reflected in highly-dense areas. Particularly, bones have a high density of matter; therefore sound waves are reflected whenever they reach the bones, generating dark areas in the ultrasound image. In lung's echography, ribs are represented as relatively broad and parallel dark areas. The image regions corresponding to intercostal spaces contain medium-intensity pixels; which are grouped into nearly parallel lines (called ' $A$ ' lines), and transversal to ribs. These lines depict the attenuation of sound waves once they traverse the pleural line (highintensity line on top) and go deeper into the human chest. The pleural line is a membrane that entirely covers the lungs; and consequently appears as the upper boundary of the dark zone. Since the pleural line is not as dense as bones, part of the sound wave is transmitted beyond the membrane, while another part is reflected, generating an echo which is represented as the medium-intensity lines as one can see in Figure 1.

Having described the structure of the image $\left(I_{m x n}\right)$, we apply a filter to remove all noise caused by both the epidermis and muscle tissue. For this purpose, the first step is to apply a binarization process of the image under a certain threshold $\left(U_{0}\right)$. The resulting matrix $B_{m x n}$ represents the binarized image on which all future transformations are performed.

$$
B_{i j}=\left\{\begin{array}{lll}
0 & \text { if } & I_{i j}<U_{o} \\
1 & \text { if } & I_{i j} \geq U_{o}
\end{array}\right.
$$

Afterwards, analysis units are set within the image. These are rectangular regions with a height equal to that of the original image $(m)$, and a width $\left(n^{\prime}=\mathrm{n} / \mathrm{d}\right)$ which is adjusted depending on the results. These regions sweep the entire image along its width.

$$
B_{\operatorname{mxn}}=\bigcup_{z=1}^{d} B_{z \operatorname{mx}\left(\frac{n}{d}\right)}
$$

Each analysis unit $\left(B_{z \operatorname{mx}}\left(\frac{\pi}{d}\right)\right.$, has been divided into two subsections: $U_{z(m v) x\left(\frac{m}{d}\right)}$ and $D_{z}\left(m-m^{J}+1\right) x\left(\frac{\pi}{d}\right)$, corresponding to the top and bottom of $B_{z \max \left(\frac{\pi}{d}\right)}$ respectively.

$B_{z m x\left(\frac{n}{d}\right)}=U_{z(m /) x\left(\frac{n}{d}\right)} \cup D_{z\left(m-m^{l}+1\right) x\left(\frac{n}{d}\right)}$

Once these subsections are defined, the total contribution of the pixels within the upper $\left(\mathrm{SU}_{\mathrm{z}}\right)$ and lower $\left(\mathrm{SD}_{\mathrm{z}}\right)$ subsections is calculated.

$$
\begin{gathered}
S U_{z}=\sum_{r=1}^{m} \sum_{s=1}^{\frac{\pi}{d}} U_{z}(r, s) \\
S D_{z}=\sum_{r=m-m^{r}+1}^{m} \sum_{s=1}^{\frac{n}{d}} D_{z}\left(r_{s} s\right) \\
E_{z}=\left|S U_{z}-S D_{z}\right|
\end{gathered}
$$

The error function $E_{\bar{\sigma}}$ is defined as the norm of the difference between the contributions of the two sub regions $\left(U_{z} y D_{\bar{z}}\right.$ ). We seek a point at which this difference is less than a certain threshold $\left(T_{0}\right)$, while keeping similar contributions from both sub regions. For this reason, the boundary between the two regions moves along the image height. Moreover, most ultrasound images display shadows at their lower part, and therefore the boundary dividing the subsections tends to move towards the top.

$E_{z} \leq T_{0} \Rightarrow\left(m^{g}, \frac{k^{*} n}{2 d}\right)$ is conserved where $\mathrm{k}=1,2,3, \ldots, \mathrm{d}$
$E_{z}>T_{0} \Rightarrow B_{z \operatorname{mx}\left(\frac{n}{d}\right)}=U_{z}\left(m^{n}-1\right) x\left(\frac{m}{d}\right) \cup D_{z}\left(m-m^{n}+2\right) x\left(\frac{m}{d}\right)$

These steps are performed in the entire width of the image. The pixels' locations that satisfy the condition set for our error function are stored in a variable $X$ as it is shown below:

$$
X=\left\{\left(B_{x_{1},} B_{y_{1}}\right) ;\left(B_{x_{2}}, B_{y_{2}}\right) ; \ldots,\left(B_{x_{d}}, B_{y_{d}}\right)\right\}
$$

With these data, it is possible to obtain a profile of the pleural membrane and then make an adjustment of these points to a line. The points of the fitted curve are stored in a variable $X^{s}$ :

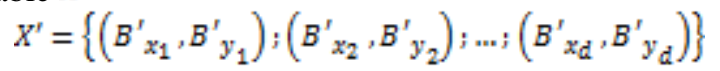

From these data, we locate the region above these points, which corresponds to the noise generated by the epidermis, muscle and other tissue. Figure 2 (a) shows both point sets: the first set or collected data $X$ (yellow dotted line) is obtained from the error function $\left(E_{\bar{z}}\right)$ applied to all subsections $U_{\varepsilon} y D_{v}$; and the second is the curve 
corresponding to the straight line $X^{t}$ (red dotted line), that best fits the previous collected data.

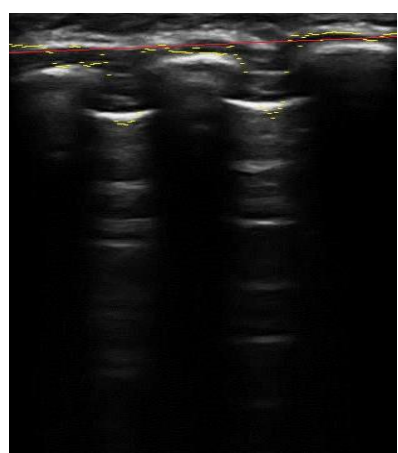

(a)

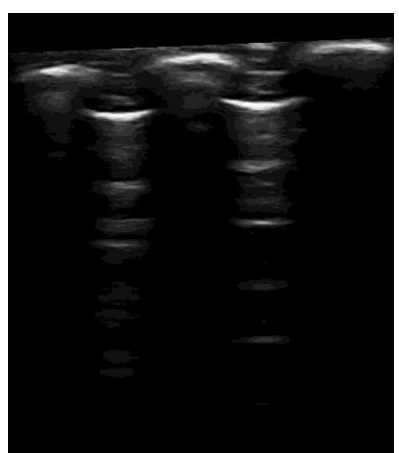

(b)
Figure 2: (a) Location of the boundary points, (b) Removing the skin on the image and replace it with pixels equal to 0

Once the first step is applied, we focus on encapsulating regions containing ' $A$ ' lines. For this, we apply to each pixel of the image $\left(I_{i j}\right)$ an intensity-dependent transformation $(T)$, that transforms intensities from the range [low in, high in] to the range [low out, high out]:

$$
G_{i j}=T\left(I_{i j}\right)
$$

With the image obtained after the transformation $T$, we proceed to its binarization with a new threshold $U_{l}$.

$$
B_{i j}^{\prime}=\left\{\begin{array}{lll}
0 & \text { if } & G_{i j}<U_{1} \\
1 & \text { if } & G_{i j} \geq U_{1}
\end{array}\right.
$$

We continue on the binarized image with a morphological operation called close $\operatorname{method}(\bullet)$. This operation allows us to smooth the contours and eliminate small holes in the image $\left(B_{\operatorname{mxn}}^{\prime}\right)$. The $B$ "' image is obtained by closing the image $B_{m x n}^{s}$ by the structural element $S E_{p q}$ : $B_{m x n}^{\prime \prime}=B_{m x n}^{r} \cdot S E_{p q}=\left(B_{m x n}^{r} \ominus S E_{p q}\right) \oplus S E_{p q} \quad \ldots$ (9)

The closing operation can be further decomposed into erosion and dilation operations. Specifically, it is defined as the erosion of $B_{\operatorname{mxn}}^{r}$ by $S E_{p q}$, followed by a dilation of the previous result with $S E_{p q}$, as it is shown in equation (Eq. 9).

Where the erosion operator is defined as:

$$
\left[B^{0} \ominus S E\right]\left(i_{v} j\right)=\min _{(g, t) \in S E}\left\{B^{0}\left(i+s_{v} j+t\right)-S E\left(s_{v} t\right)\right\}
$$

Likewise, the dilation operator is defined as:

$$
\left[B^{\prime} \oplus S E\right]\left(i_{i} j\right)=\max _{(s, t) \in S E}\left\{B^{s}\left(i-s_{v} j-t\right)+S E\left(s_{v} t\right)\right\}
$$

The image $B_{m x n}^{\prime \prime}$ (Figure 3 (a)) looks to emphasize the shapes, homogenizing objects located in the image. Subsequently, edge detection $(S)$ of the objects contained in the image is performed, by applying the Sobel method:

$$
B^{m x}{ }_{m x}=S\left(B^{H}{ }_{m x n}\right)
$$

From the image $B^{m x}{ }_{m x n}$ (Figure 3 (b)), an structural element $C_{a b}$ of rectangular shape is defined, with height equal to 5 pixels and width equal to that of the original image $(n)$, which will be define the boundaries of the ' $A$ ' lines.
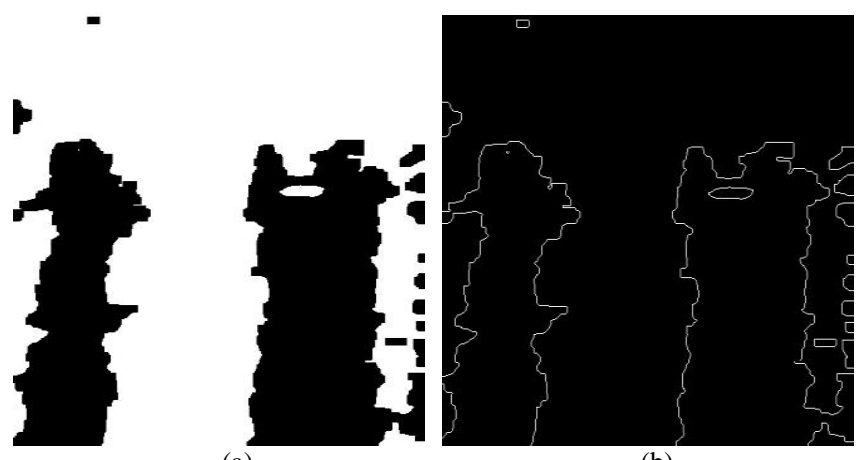

(b)

Figure 3: (a) Aplication close on the image B', (b) Aplication edge detection on the image B',

The locating our structural element $C$ will depend on criteria found from the same ultrasound image. If image has the presence of pixels with high-intensity value in the deepest area, the structural element will be located between the bottom of the pleural line and the deepest area (pleural line $X$ is taken according to approach detailed above). But, in the opposite case, the location of the structural element will be in the top third of the image from the pleural line $X^{\prime}$. Once the structural element $(C)$ is located, the average of pixels values of all columns $(n)$ is calculated:

$$
C_{1 x m}^{t}=\sum_{i=1}^{5} \frac{C_{i x n}}{5}
$$

Then we store both initial position as the end of each different neighborhood of zero vector $C_{n}^{\gamma}$ in a new vector $\ln d_{g}(s \leq n)$.

With the above result, we extract these regions in the image, resulting from the extraction a first approximation, the noise of the skin, and these regions would conform our new set $Y$.

$$
Y=\left\{B_{m x}(\operatorname{Ind}(1): \operatorname{Ind}(2)), \ldots, B_{m x}(\operatorname{Ind}(s-1): \operatorname{Ind}(s)\}\right.
$$

$$
a: b=\text { calling } a \text { vector from the index } a \text { to } b
$$

The last step is performed on each element of the set. It consists to define an object $G$ of width equal to that of each set member and height equal to 5 pixels. This object sweeps the area between the bottom of the curve to the pleura $\left(X^{f}\right)$ to some degree of depth $A$.

$$
X^{H}=\max \left(\bigcup_{m=\max }^{\max \left(X^{n}\right)} \sum_{i=\mathrm{m}}^{\operatorname{mon}} \sum_{j=1}^{m+A} G_{i j}\right)
$$

\section{Where}

$h=$ width of each element of the set $Y$

$A=\left(m-\max \left(X^{t}\right)\right) / 3$ 
This step is obtained from an adjustment in the location of the pleural area $X^{n t}$ the resulting image is shown in Figure 4 (a): (a)

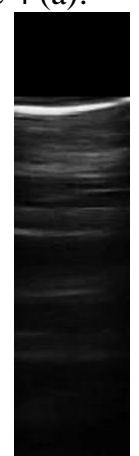

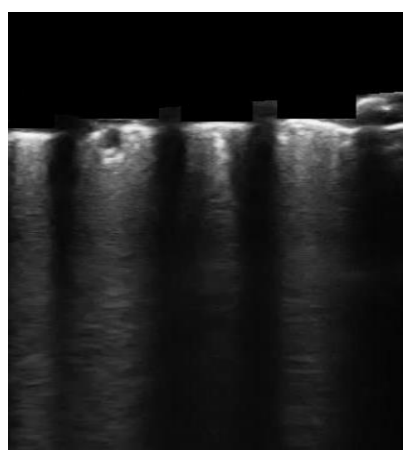

(b)
Figure 4: (a) Extraction of the pleural zone, (b) Image without skin

Following the steps already discussed, we obtain the location of the pleural line, and consequently, it is possible to remove of the noise generated by the skin, muscles and other tissues (Figure 4 (b)).

\section{Evaluation:}

44 frames (20 with evidence of pneumonia and 24 with no evidence of pneumonia, classified by the human expert), were analyzed with our algorithm to remove the skin portion. In addition, for all the 44 frames, the human expert removed the skin portion manually. Using software of image edition, the human experts manually draw the pleural line that limits the skin portion. From each frame, the central section between the acoustic shadows from the rib was considered for the analysis.

The pleural line obtained by the algorithm was compared with the pleural line manually drawn by the human expert.

\section{RESULTS AND DISCUSSION}

For 43 of the 44 frames, the pleural line estimated by the algorithm was very close to the pleural line draw by the human expert. The mean quadratic error was 11.17 pixels $(\min =7.17, \max =13.67, \mathrm{STD}=1.57)$. In all these cases the estimated pleural line was over the manually draw line. Therefore, in any case the algorithm erroneously removed evidence of pneumonia. In one frame, the estimated pleural line appeared more distant (mean quadratic error $=76.37$ pixels) from the manually draw. This estimated line appeared closer to the skin surface, due to noise intense lines in the skin portion.

The resulting images obtained after applying the specific processing described here are characterized for containing regions with high intensity that could either be the normal " $A$-lines" (Figure 1) observed in healthy lungs or pneumonia. The encapsulation and preservation of these regions play a significant role in our algorithm. We found that the presence of the ribs, which are irregular areas, makes difficult the identification and correct removal of skin and other tissues, which is the primary objective in this work.
The methodology presented here, corresponds to a sequential programming algorithm rather than an objectoriented programming (OOP) architecture. The improvement of the programming architecture is an objective that we plan to develop in the future.

One aspect to highlight in the development of the code is the threshold establishment for the binarization process $\quad\left(U_{0}=45 y U_{1}=16\right), \quad$ the module $\left|S U_{z}-S D_{z}\right|<T_{0} \quad\left(T_{0}=15\right)$, and the top of our structural element $C_{5 x n}$. These values were manually optimized through the multiple tests executed at each stage of code. Further studies for automatic optimization are planned to be conducted.

\section{CONCLUSIONS}

- The digital noise associated to the skin and external tissues upwards the ribs present in ultrasound images of lungs, was significantly removed by our algorithm.

- The elimination of the skin portion in lung ultrasound images facilitates the interpretation and positively contributes in automatic recognition of pneumonia.

- This method could be the starting step for automatic recognition of lung consolidates during the diagnostics of pneumonia in children based on lung ultrasound images.

\section{Acknowledgements}

This work was supported by NIH-1D43TW009349-03, Grand Challenge Canada 0542-01-10, Grand Challenge Canada 0688-01-10, CONCYTEC-FONDECYT 0542014, PUCP-DGI 70242-2149, and 1-2013-Fondecyt.

\section{REFERENCES}

[1] UNICEF. "Pneumonia and Diarrhoea: Tackling the deadliest diseases for the world's poorest children. New York: UNICEF, 2012." (2013).

[2] M. Pereda, M. Chavez, C. Hooper-Miele, R. Gilman, M. Steinhoff, L. Checkley. "Lung Ultrasound for the Diagnosis of Pneumonia in Children: A Meta-analysis", Pediatrics, 135(4), 714-722, 2015

[3] Xie, Jun, Yifeng Jiang, and Hung-tat Tsui. "Segmentation of kidney from ultrasound images based on texture and shape priors." Medical Imaging, IEEE Transactions on 24.1 (2005): 45-57.

[4] Gehmacher, Otto. "Ultrasound pictures of pneumonia." European Journal of Ultrasound 3.2 (1996): 161167.

[5] Reissig, Angelika, et al. "Lung ultrasound in the diagnosis and follow-up of community-acquired pneumonia: a prospective, multicenter, diagnostic accuracy study." CHEST Journal 142.4 (2012): 965-972.

[6] Reissig, Angelika, and Roberto Copetti. "Lung ultrasound in community-acquired pneumonia and in interstitial lung diseases." Respiration 87.3 (2014): 179-189.

[7] Patrícia, S. de A., Hugo da C. Ribeiro, and Carlos Maurício C. Mendes. "Temporal trends of overall mortality and hospital morbidity due to diarrheal disease in Brazilian children younger than 5 years from 2000 to 2010." Jornal de pediatria 89.3 (2013): 315-325. 